prevailing design there following the competition to rebuild the Hôtel-Dieu in Paris after the disastrous fire of 1772. Florence Nightingale supported pavilions, advocating hospital wards with large windows between every two beds, but criticized the design of the Royal Vic, where she felt that nurses on the wards would struggle to observe their patients efficiently.

Adams explores the important contribution of Edward Stevens, arguably the first specialist architect of hospitals. Stevens trained at Massachusetts Institute of Technology and made rigorous studies of European and North American hospitals and the practice of medicine. He designed, with Frederick Lee, numerous hospitals across the United States and Canada and wrote the influential guide The American Hospital of the Twentieth Century in 1918. Also influential were physician consultants such as $\mathrm{S}$.
S. Goldwater, the superintendent of New York Hospital. Goldwater proposed a design in 1905 for an urban high-rise hospital exploiting the new technologies of structural steel, elevators and electric lights. Although never built, the proposal was quickly followed by numerous designs for such multi-storey hospitals.

With medicine progressing ever faster, Adams' history reminds us why hospital architects and physicians should work together to optimize healthcare environments.

D. Kirk Hamilton is an associate professor of architecture, and a fellow and associate director of the Center for Health Systems \& Design at the College of Architecture, Texas A\&M University, College Station, Texas 77843-3137, USA. He is the co-editor of Health Environments Research \& Design Journal.

\title{
Quest for extraterrestrial life
}

\author{
The Living Cosmos: Our Search for Life in \\ the Universe \\ by Chris Impey \\ Random House: 2007.416 pp. $\$ 27.95$
}

\section{Bruce Jakosky}

From only one example, that of life on Earth, we have learned a lot about what makes a planet habitable. We know how life functions, how it may have originated and some types of environment that support its existence. Yet we do not know how widespread life might be. To do so we must extend our knowledge past Earth, into the Solar System and beyond.

The possible existence of extraterrestrial life - be it in the form of microbes on Mars, biological forms on planets orbiting other stars, or, frankly, UFOs and aliens visiting Earth - is fascinating. Yet astrobiology is firmly science-based rather than speculative and aimed at a curious public. Its major questions can be answered empirically with appropriate spacecraft and telescopes. To elucidate the origins of life, we might ask for example, is Mars habitable? Can signs of life be found there or on moons such as Jupiter's Europa and Saturn's Titan? Are there Earth-like planets with environments conducive to life around other stars? Might there be civilizations elsewhere in our Galaxy? If so, could we listen in on their conversations?

These questions run through The Living Cosmos. Astronomer Chris Impey provides a broad, accessible context for his thoughtful, engaging and up-to-date take on the quest for extraterrestrial life. The start and the end of the book pose questions about the relationship between art and science, and the likelihood of extraterrestrials having either. The rest dwells mainly on the science. Starting with the historic foundations of the field during the copernican revolution and following the story through modern science's development, key chapters deal with the origins of life, life in extreme environments and evolution here on Earth. The story then moves out into the Solar System and planets around stars other than our Sun. A far-reaching final chapter tackles intelligent life, interstellar travel, and the meaning of the search for life. Impey details current and future space missions and extrasolar planet research.

How is this exploration progressing and what lies ahead? Mars is arguably the best place to look for present or past life, given the evidence for liquid water there and the planet's proximity to Earth. Although many spacecraft have been sent, the future of the programmes is uncertain. Five craft currently send back data: the exploratory robot rovers Opportunity and Spirit from its surface, and orbiters Mars Odyssey, Mars Reconnaissance and Mars Express. In the United States, the next missions are over budget or delayed, notably the Mars Science Laboratory (scheduled for a 2009 launch) and the Mars Scout orbiter (now scheduled to launch in 2013). Despite repeated calls for a Mars Sample Return, plans for that or other missions beyond 2013 are unclear, especially in light of the recently released proposed budget for next year.

Beyond Mars, several moons in the outer Solar System are worth exploring. Jupiter's satellites Europa, Ganymede and Callisto are good targets because they may have recently hosted liquid water. Saturn's moons Titan and Enceladus, targets of the ongoing Cassini mission, are of great interest because of the abundance of organic molecules (especially on Titan) and the potential for near-surface liquid water (Enceladus). NASA is studying possible missions to these objects as a prelude to a major push mission into the outer Solar System.

To explore these remote places and perform science there, we need new technologies. Returning a sample of martian rocks and soil involves lifting the matter off Mars' surface, sealed tightly enough to avoid releasing any captured organisms into the terrestrial environment, and then flying the capsule back to Earth. Spacecraft must survive harsh radiation fields around the giant planets and perform difficult manoeuvres deep within their gravitational wells to orbit or land on a satellite.

These problems are solvable. But in our tight-budgeted times, technology development is often of lower priority than maintaining ongoing missions and starting new ones. Ironically, having the new technology in hand at the start of mission development helps prevent budget overruns later on. It is shortsighted to skimp on innovation. We pay the costs later, possibly in axed missions.

Recently, insufficient funding has postponed key astrobiology missions such as Terrestrial Planet Finder and Mars Sample Return. NASA and the science community must work together to address cost overruns and optimize research within the funding available. Once we have a viable plan of action, we will be able to answer the questions that Impey has ably outlined, and continue the search for life elsewhere.

Bruce Jakosky is professor of geological sciences at the Laboratory for Atmospheric and Space Physics, University of Colorado, Campus Box 392, Boulder, Colorado 80309-0392, USA. He is the author of Science, Society, and the Search for Life in the Universe.

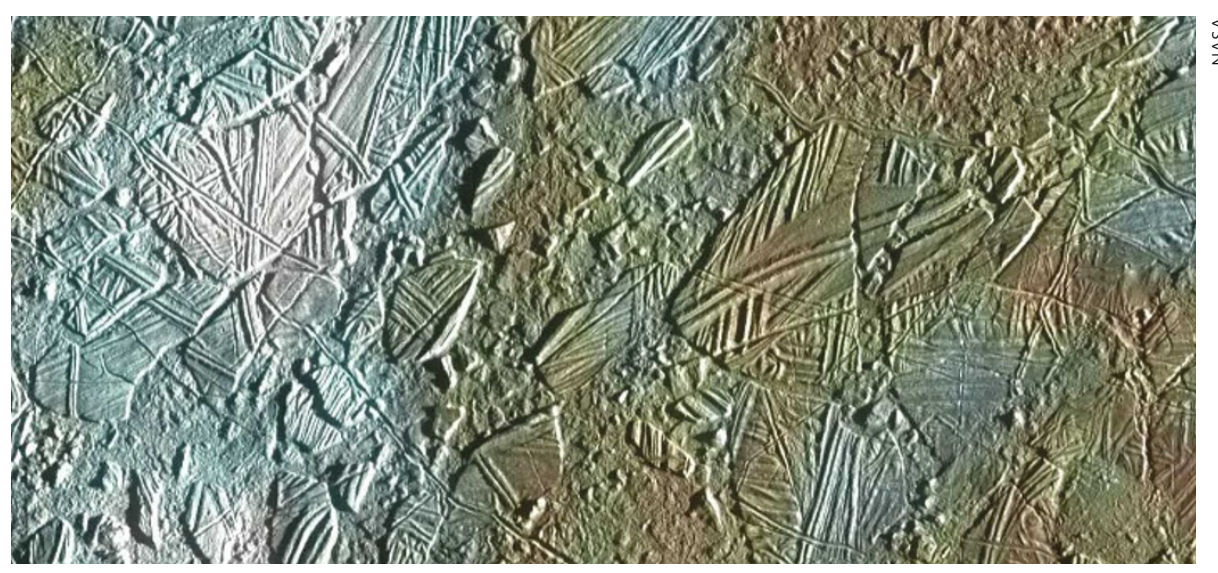

The icy surface of Jupiter's moon Europa may recently have hosted water: could it support life? 